\title{
Multiplicador del Gasto Público en Argentina*
}

\author{
Jorge Pablo Puig ${ }^{1}$
}

(UNLP)

Esta Versión: Abril 2014

\section{Resumen}

Este trabajo proporciona por primera vez estimaciones empíricas sobre el multiplicador del gasto del Sector Público Nacional de Argentina, utilizando una metodología de vectores autorregresivos estructurales (SVARs). Al mismo tiempo, producto de contar con la clasificación económica del gasto realiza una contribución más que interesante diferenciando el multiplicador según se trate de gasto en consumo o gasto en capital (o inversión pública). Los resultados muestran la enorme importancia de dicha diferenciación en cuanto el gasto el consumo tiene efectos moderados sobre el producto, mientras que la inversión pública tiene efectos macroeconómicos mucho más expansivos, producto de las sinergias que la misma genera con el sector privado. A su vez van en línea con resultados previos para otros países en desarrollo y son consistentes con el resultado esperado dada la tradicional prociclicidad de la política fiscal del país.

Se pone de manifiesto la importancia de pensar el sector público y el privado como complementos más que como sustitutos (o rivales) y la necesidad de abandonar las políticas fiscales procíclicas.

Palabras Claves: Multiplicador, Política Fiscal, Ciclo Económico, SVAR.

Clasificación JEL: E32, F62.

* Este trabajo constituye la tesis final de la Maestría en Economía de la Universidad Nacional de La Plata (UNLP) realizada bajo la dirección de Guillermo Vuletín y la co-dirección de Ricardo Bebczuk. El autor agradece inconmensurablemente su apoyo. Los errores u omisiones son de su exclusiva responsabilidad.

${ }^{1}$ Universidad Nacional de La Plata. Email:jorgeppuig@gmail.com 


\section{Introducción}

En su forma básica, se conoce como multiplicador fiscal al cambio en la actividad económica ante un cambio en algún instrumento de política fiscal como el gasto del gobierno, los impuestos o las transferencias.

Conocer su tamaño es importante para los gobiernos ya que un multiplicador alto indica que la política fiscal tiene grandes efectos sobre la economía real, lo que no sólo significa que expandir el gasto es una medida eficiente para impulsar la economía, sino que también los gobiernos deben ser cuidadosos al considerar la implementación de medidas de austeridad fiscal. En ese caso, recortes en el gasto o aumentos de impuestos para equilibrar los presupuestos públicos pueden tener efectos perjudiciales sobre la economía. Por el contrario, si el multiplicador es muy bajo, los gobiernos pueden tener menos incentivos para ceder ante las demandas de recortes de impuestos o aumentos de gasto dado que la política fiscal no tendrá un efectos significativos sobre la economía. Por estas razones, es útil para los gobiernos saber cuán eficiente son sus políticas fiscales.

La última crisis económica acontecida a nivel mundial ha forzado a que muchos países implementaran paquetes de estímulo o medidas de austeridad fiscal. ${ }^{2}$ Como consecuencia, una de las preguntas centrales en la política macroeconómica de los últimos años ha sido: ¿Cuál es el efecto del gasto del gobierno sobre la actividad económica? Así, las discusiones sobre el tamaño de los multiplicadores han resurgido dentro de la literatura en conjunto con un esfuerzo creciente para encontrar valores precisos de ellos. Sin embargo, tanto desde un punto de vista teórico como empírico se da cuenta de un amplio rango de multiplicadores. Por ejemplo, por el lado del gasto público el multiplicador se ubica en un rango que va desde -2.3 (en países altamente endeudados según Ilzetzky, Mendoza y Vegh, 2010) hasta 3.6 (durante las recesiones según Auerbach y Gorodnichenko, 2011).

¿A qué se deben estas diferencias? Una razón puede encontrarse en que éstos son propensos a depender de las características de la economía en cuestión, como por ejemplo el grado de apertura, el régimen de tipo de cambio y la fase del ciclo económico. ${ }^{3}$ Este último factor aparece particularmente como relevante si se adoptan los argumentos keynesianos tradicionales destacando que el gasto del gobierno es probable que tenga mayores efectos expansivos durante las recesiones que en las expansiones. Intuitivamente, cuando la economía enfrenta malos tiempos, las expansiones del gasto público son menos propensas a desplazar el consumo privado o la inversión. Así, será determinante para el tamaño del multiplicador la ciclicidad que muestre la política fiscal, tema que fue largamente estudiado en la literatura logrando consenso acerca de la presencia de políticas fiscales contracíclicas (ej. expansiones de gasto en malos tiempos) en países desarrollados y procíclicas en países en desarrollo (ej. expansiones de gasto en épocas de bonanza). ${ }^{4}$ Así, en el marco de la teoría keynesiana es de esperar multiplicadores mayores en el primer grupo de países. En este contexto, Argentina que pertenece al grupo de países en desarrollo y son extensas las contribuciones que destacan su procíclica fiscal en las últimas décadas debería presentar, en principio, un multiplicador modesto. ${ }^{5}$ Otra explicación de la diferencia en la magnitud de los multiplicadores proviene desde el punto de vista metodológico dado que su estimación no resulta una tarea sencilla y está sujeta a una serie de problemas. El mayor de ellos es el de endogeneidad en el análisis de causalidad de la política fiscal: por un lado, la política fiscal afecta a la economía real a través de múltiples canales (ej.

\footnotetext{
${ }^{2}$ Véase Cwik y Wieland (2011) para una discusión con mayor detalle.

${ }^{3}$ Véase Ilzetzki, Mendoza y Vegh (2010) y Auerbach y Gorodnichenko (2012).

${ }^{4}$ Véase Gavin y Perotti (1997), Braun y Di Gresia (2003), Kaminsky, Reinhart y Vegh (2004), Talvi y Vegh (2005) y Frankel, Vegh y Vuletin (2011).

${ }^{5}$ Véase Braun y Di Gresia (2003), Porto (2004), Díaz Frers (2010) y Frankel, Vegh y Vuletín (2011).
} 
los gobiernos pueden aumentar los salarios, elevar el poder adquisitivo y generar un aumento el consumo total). Por otro lado, la economía real también influye en la política fiscal (ej. en los buenos tiempos económicos, los residentes pagan más impuestos, por lo tanto, aumentan los ingresos del gobierno y los aumentos de gasto se vuelven más factibles).

Por lo tanto, es fundamental hacer una distinción entre políticas fiscales endógenas y exógenas. Las primeras hacen referencia a las reacciones automáticas de los gastos y los ingresos del gobierno debido al ciclo económico. Las segundas a las medidas de política que no son consecuencia de la situación actual de la economía. Para calcular multiplicadores fiscales, los encargados de formular políticas están exclusivamente interesados en los efectos de éstas últimas y de ahí la necesidad de una estrategia de identificación de dichos efectos para calcular multiplicadores fiscales (Blanchard y Perotti, 2002). Dos estrategias son las más tradicionales en la literatura.

Siguiendo a Blanchard y Perotti (2002), la primera estrategia se basa en la estimación de un modelo estructural de vectores autorregresivos (SVAR) en combinación con el uso de datos trimestrales. ${ }^{6}$ Estos autores identifican cambios en la política fiscal utilizando información proveniente de instituciones fiscales. Por el lado del gasto, su supuesto de identificación consiste en que el gasto del gobierno requiere al menos un trimestre para responder a las cambios en el producto. Por el lado de los impuestos, usan la elasticidad-producto de ellos para aislar el componente cíclico de la recaudación y tratar de captar así los cambios exógenos en la política tributaria. De este modo, argumentan que una vez corregido el impacto de la actividad económica en los ingresos y se controla el comportamiento de gasto público, el uso de datos trimestrales elimina virtualmente cualquier preocupación con respecto al cambio de la política fiscal en respuesta a las fluctuaciones inesperadas en la actividad económica durante ese trimestre.

La segunda estrategia se basa en lo que se conoce como "experimentos naturales". Iniciada por Barro (1981), identifica cambios exógenos en la política fiscal a través del uso de variables o instrumentos que no correlacionan con el ciclo económico. Por el lado del gasto los estudios se han centrado típicamente en determinadas categorías, como los gastos militares o el gasto para afrontar catástrofes naturales, que son menos propensos a responder a las fluctuaciones del producto. ${ }^{7}$ Por el lado de los impuesto se recurre, por ejemplo, a información sobre cambios en las alícuotas impositivas en conjunto con información legislativa y periodística para captar los cambios exógenos en la política tributaria. Se argumenta que la alícuota (en contraposición a la base imponible) es un instrumento (y no un resultado) sobre el cual el hacedor de política puede actuar independientemente del ciclo económico. ${ }^{8}$

Ambas técnicas han sido ampliamente utilizadas en las últimas décadas para estimar multiplicadores fiscales en países desarrollados como Estados Unidos, Alemania y aquellos que integran la Organización para la Cooperación Económica y Desarrollo (OCDE). Aunque con menor intensidad, a partir de la última crisis mundial han proliferado los estudios para países menos desarrollados, varios de ellos pertenecientes a América Latina a excepción de Argentina.

Por tal motivo este estudio se propone contribuir a la literatura con las primeras estimaciones empíricas específicamente para Argentina sobre el multiplicador del gasto del Sector Público Nacional, aplicando una estrategia de identificación perteneciente al primer grupo de los mencionados

\footnotetext{
${ }^{6}$ Véase, por ejemplo, Perotti (2004) Favero y Giavazzi (2007), Mountford y Uhlig (2009), Favero y Giavazzi (2010), Gordon y Krenn (2010), Ilzetzki, Mendoza y Vegh, (2010) e Ilzetzki (2011).

${ }^{7}$ Véase, por ejemplo, Barro (1981), Ramey y Shapiro (1998), Hall (2009), Ramey (2011) y Barro y Redlick (2011).

${ }^{8}$ Véase, por ejemplo, Ramey (2011), Hall (2009), Barro y Redlick (2011), Romer y Romer (2010) y RieraCrichton, Vegh y Vuletin (2012).
} 
anteriormente. Se estima aquí un SVAR con datos trimestrales para el período comprendido entre el primer trimestre de 1993 y el cuarto de 2012. Adicionalmente, producto de contar con la clasificación económica del gasto, se realiza una contribución más que interesante al diferenciar el multiplicador del gasto público según se trate de gasto en consumo o gasto en capital (o inversión pública) al tiempo que se analizan los efectos sobre el resto de los componentes de la demanda agregada. Realizar este punto, sin dudas, resulta muy útil a la hora de evaluar el poder del gasto para influir sobre el nivel de actividad, explorando tanto la relevancia de su composición como la posible existencia (o no) de sinergias entre la inversión pública con la inversión privada. Esto último daría indicios ciertos sobre la articulación entre el sector público y el privado en cuanto a si existe algún tipo de complementariedad entre ambos o si de lo contrario son rivales.

Tanto la elección de la estrategia de identificación como la no estimación del multiplicador de los impuestos se debe a dos motivos. Primero, un país como Argentina no registra algo similar al gasto militar o al gasto ante desastres naturales que registre peso significativo en el presupuesto y que sea utilizable como instrumento para medir cambios exógenos en la política fiscal. Segundo, aportes recientes a la literatura sostienen que el problema de endogeneidad es menos plausible de solución vía el SVAR por el lado de los impuestos que del gasto. En concreto, aún utilizando la medida de ingresos ajustados por el ciclo económico resulta probable que dicha medida de política fiscal continúe presentando cierta vinculación a la actividad económica por otros canales. ${ }^{9}$ El supuesto por el lado del gasto respecto que los shocks de política fiscal causan efectos cuando son implementados y no cuando son anunciados, goza de mayor aceptación en la literatura. Considerando lo anterior, los resultados hallados aquí pueden utilizarse como una estimación de referencia para nuestro país y como instrumento para la comparación internacional, proporcionando información sobre los efectos de la política fiscal en Argentina.

Los principales resultados hallados se pueden resumir de la siguiente manera:

1. El multiplicador del gasto público en Argentina es bajo y se sitúa en $\$ 0.18$ al cabo de 8 trimestres. Esto significa que el aumento de $\$ 1$ en el gasto del gobierno nacional, aumenta el producto en $\$ 0.18$ transcurrido dicho horizonte temporal y pone de manifiesto la existencia de efectos desplazamiento sobre la producción.

2. A la hora de diferenciar los multiplicadores del gasto según su clasificación económica, el multiplicador del gasto en consumo se encuentra muy por debajo del multiplicador del gasto en capital (o inversión publica). El primero alcanza también un valor de $\$ 0.18$ y esto se explica dado que el gasto en consumo representa más del $90 \%$ del gasto total. El multiplicador del gasto en capital es sistemáticamente mayor a la unidad.

3. Cuando se analizan los canales de transmisión del gasto (sus efectos sobre el resto de los componentes de la demanda agregada) se encuentran multiplicadores positivos del gasto sobre el consumo privado y sobre la inversión privada, lo que da indicios de la existencia de sinergias entre el sector publico y el privado en materia de inversión. El multiplicador sobre las exportaciones netas resulta negativo aunque sin soporte estadístico.

En lo que resta, el trabajo se organiza de la siguiente manera. La Sección 2 proporciona una revisión general de la literatura académica existente tanto a nivel teórico como empírico. La Sección 3 presenta la metodología empleada para estimar los multiplicadores del gasto mientras que los datos

\footnotetext{
${ }^{9}$ Para una discusión más detallada sobre este punto véase Riera-Crichton, Vegh y Vuletin (2012).
} 
utilizados se describen en la Sección 4. La Sección 5 presenta los resultados para la especificación de referencia basada en la estimación de SVARs de dos variables y la Sección 6 hace lo propio con una extensión al caso de tres variables. Por último, los comentarios finales la Sección 7 concluyen.

\section{Revisión de la literatura}

Como se mencionó en la sección anterior calcular el multiplicador fiscal no es una tarea sencilla ya que ninguna técnica para su cálculo o estimación es perfecta. En esta sección se propone realizar una revisión de las cuestiones teóricas que determinan el multiplicador como así también de la literatura empírica existente hasta el momento.

\subsection{Los mecanismos teóricos y sus predicciones}

Como en muchas otras ramas de la teoría económica, los supuestos sobre el comportamiento del agente económico son el cruciales. Una de las discusiones más importantes en la literatura se da sobre si los agentes tienen o no la capacidad de dejar que sus decisiones en el presente sean influenciadas por las expectativas racionales formadas en base a lo acontecido en el pasado, el comportamiento presente y el futuro.

\subsubsection{Sin expectativas}

En el modelo clásico, se asume plena flexibilidad de precios y salarios, y la curva de oferta es completamente vertical. Esto implica que cualquier política fiscal es totalmente absorbida por la economía a través de los niveles de precios. Por lo tanto, no hay efecto en la producción, porque el nivel de producto es determinado por la demanda (Mankiw y Taylor, 2008).

Por otro lado en los modelos keynesianos, los precios son rígidos, las empresas pueden comportarse estratégicamente y existe desempleo. ${ }^{10}$ Ante la ausencia de expectativas, el consumo depende solamente de los ingresos período actual. Esta configuración permite que la política fiscal pueda tener un efecto sobre el producto y el mecanismo exacto depende de las características de la economía nacional en cuanto a su nivel de empleo y de apertura.

En una economía cerrada, el efecto directo de un aumento del gasto público se traduce en un aumento del producto directamente a través de las compras del propio gobierno. Pero a su vez un impulso en el gasto también tiene efectos indirectos, dado que los gastos del gobierno son ingresos para los agentes económicos que venden sus productos al gobierno. Así un aumento de gasto es por lo tanto también un shock de ingresos, que conduce a mayores gastos de los destinatarios de ellos lo que a su vez conduce a mayores ingresos para otros agentes y así sucesivamente. El efecto total de ese impulso en el gasto sobre el producto es el multiplicador que se estima en este trabajo y mayor será su magnitud cuanto menor sea el nivel de empleo en la economía dado que habrá mas espacio para una reactivación.

En una economía pequeña con fronteras abiertas y un tipo de cambio flexible, los aumentos del gasto elevan la tasa de interés, lo que aumenta la demanda de moneda local, llevando a una apreciación nominal. Como los precios son rígidos y el capital móvil internacionalmente, el tipo

\footnotetext{
${ }^{10}$ Modelos keynesianos pueden ser vistos dentro de un marco tradicional Mundell-Fleming con fricciones de precio. Véase, por ejemplo, Mankiw y Taylor (2008).
} 
de cambio real se aprecia. Esto conduce a una disminución en las exportaciones, neutralizando el impulso inicial positivo en el producto. Por el contrario si el tipo de cambio está fijo, la apreciación es prevenida con el aumento de la oferta monetaria y el producto puede crecer. En otras palabras, el multiplicador es positivo.

Si la expansión del gasto se financia vía endeudamiento en exceso y existe la posibilidad de provocar un aumento de las tasas de interés, puede conducir a la reducción de la inversión privada o lo que se conoce como efecto desplazamiento del gasto sobre la inversión. La magnitud del efecto desplazamiento así como el multiplicador depende de la velocidad de ajuste de los precios nominales. Cuanto más rígidos estos, menor el efecto desplazamiento, y mayor el multiplicador. ${ }^{11}$

De cualquier modo, vale aclarar aquí que aunque el multiplicador en un marco keynesiano es generalmente positivo, hay situaciones en que pueden revelarse multiplicadores cercanos a cero e incluso negativos. Si consideramos la ecuación tradicional del producto $\mathrm{Y}=\mathrm{C}+\mathrm{I}+\mathrm{G}+\mathrm{XN}^{12}$ , y suponemos que $\mathrm{G}$ aumenta en 3 unidades, $\mathrm{XN}$ y $\mathrm{C}$ disminuyen también en 1 debido a una apreciación del tipo de cambio e I se reduce en 1 unidad debido al efecto desplazamiento, entonces el efecto total sobre Y es nulo. De ahí la importancia de las características de la economía nacional y sus vínculos con el resto del mundo (Mankiw y Taylor, 2008; Spilimbergo, Symansky y Schindler, 2009 e Ilzetzki, Mendoza y Vegh, 2010).

\subsubsection{Con expectativas}

Ahora bien si los agentes tiene la capacidad de mirar hacia adelante, la situación cambia considerablemente y también gana en complejidad. Los modelos económicos simples como el marco de Mundell-Fleming son menos adecuados para tratar las expectativas, por lo que se necesita la implementación de modelos de equilibrio general estocástico y dinámico (DSGE) que por lo general han confiado en las especificaciones neoclásicas.

Estos modelos, conocidos como "ciclo real de negocios" (RBC), descansan en el supuesto de que los precios son totalmente flexibles y la competencia es perfecta. La previsión realizada sobre el futuro por parte de los agentes se basa en un comportamiento típicamente "Ricardiano": un aumento en el gasto del gobierno lleva a los agentes a suponer que se financiará con mayores impuestos en el futuro de modo tal de mantener el presupuesto en equilibrio. ${ }^{13}$ Como los agentes internalizan el comportamiento futuro de los gobiernos, se da un efecto de riqueza negativo en el presente, es decir, el consumo disminuye en favor del ahorro, llevando a una suba de las tasas de interés y un aumento de la oferta de trabajo que hace disminuir el salario. Más notable aún es que la disminución en el consumo es independiente de la forma en que está financiado el gasto del gobierno, dado que las expectativas son racionales. Ésta disminución en el consumo dependerá de si los shocks son temporales o permanentes. Bajo uno permanente, la caída del consumo privado equilibra totalmente el aumento en el gasto gubernamental, llevando a un multiplicador inferior. Bajo uno temporal, el consumo cae menos fuertemente y multiplicadores positivos son factibles de hallar. ${ }^{14}$

\footnotetext{
${ }^{11} \mathrm{Si}$ bien no se estimará en este trabajo el efecto del multiplicador de los impuestos tiene el mismo signo (negativo para un aumento de impuestos y positivo para un recorte) pero puede ser menor que el del gasto público, dado que parte del ingreso disponible puede ser ahorrada (Mankiw y Taylor, 2008).

${ }^{12}$ Donde Y representa el producto, C el consumo privado, I la inversión privada, G el gasto público y XN las exportaciones netas

${ }^{13}$ Del igual modo, un recorte de impuestos hace que los agentes esperen futuros recortes en el gasto o aumentos de impuestos.

${ }^{14}$ Véase Baxter and King (1993).
} 
Por otra parte el marco Nuevo Keynesiano (NK) asume rigideces nominales, competencia monopolística y desempleo. Los resultados NK se diferencian de los obtenidos bajo el marco anterior en un punto elemental: los salarios aumentan debido a una mayor demanda de mano de obra por el gasto del gobierno. Además, este efecto es mayor que la presión a la baja que origina un aumento en las horas trabajadas. Esto conduce a una respuesta positiva del consumo implicando que la política fiscal tendrá un efecto más que proporcional en la producción, lo que básicamente disminuye el esfuerzo que un gobierno tiene que hacer para sostener la actividad. Dada esta situación, los multiplicadores son generalmente positivos.

En conclusión se aprecia que según qué marco teórico (y el conjunto de supuestos en consecuencia) se utilice la predicciones difieren. Así las preguntas relevantes aquí son, entonces, si los supuestos son realistas, y en qué grado deben integrarse en un modelo para que sea una representación cercana de la realidad. ${ }^{15}$

\subsection{Resultados empíricos}

Las estrategias empíricas para calcular multiplicadores fiscales deben identificar los cambios exógenos y endógenos, es decir, de qué manera se trata el problema de endogeneidad. Como se mencionó en la introducción de este trabajo, dos son las estrategias tradicionalmente utilizadas y los principales resultados hallados a través de ellas se presentan en las subsecciones siguientes.

\subsubsection{Modelos de vectores autorregresivos estructurales (SVAR)}

Los vectores autorregresivos estructurales (SVAR) se han convertido en los más utilizados en la literatura debido a su poder de predicción y su relativa sencillez de implementación. Su estrategia de identificación supone que los cambios en el producto no pueden dar lugar a cambios en la política fiscal durante el mismo período. Es decir, que existe una demora entre que una medida fiscal es anunciada y su efectiva implementación lo que vuelve muy relevante la periodicidad de los datos a utilizar.

Utilizados originalmente para evaluar los efectos de la política monetaria, Blanchard y Perotti (2002) son los primeros en aplicar esta estrategia a la política fiscal de los Estados Unidos. Los autores estiman un modelo de tres variables (impuestos, gasto y producto) y por el lado del gasto público suponen que demora al menos un trimestre para responder a las cambios en el producto. Por el lado de los impuestos, usan la elasticidad-producto de ellos para aislar el componente cíclico de la recaudación y tratar de captar así los cambios exógenos en la política tributaria. De este modo, argumentan que una vez corregido el impacto de la actividad económica en los ingresos y se controla el comportamiento de gasto público, el uso de datos trimestrales elimina virtualmente cualquier preocupación con respecto al cambio de la política fiscal en respuesta a las fluctuaciones inesperadas en la actividad económica durante ese trimestre. Sus multiplicadores están cerca de la unidad en la especificación básica y alcanzan valores cercanos a 2, cuando se incluyen las previsiones.

Perotti (2004) amplía el modelo Blanchard y Perotti (2002) con dos variables adicionales, la tasa de interés y el nivel de precios, con el fin de controlar los posibles efectos de la política monetaria.

\footnotetext{
${ }^{15}$ Se recomienda consultar Spilimbergo, Symansky y Schindler (2009) para una revisión de las diferentes condiciones que pueden influir sobre el signo y la magnitud de los multiplicadores fiscales. La mayoría de las condiciones son bastante razonables: una mayor propensión marginal a consumir, una menor propensión a importar, consumidores no-Ricardianos o un mayor gasto en relación con todos los recortes de impuestos aumentan los multiplicadores actuando sobre el vínculo entre la política fiscal y el producto.
} 
Estima un SVAR para un grupo de 5 países de la OCDE y realiza a su vez un análisis de los componentes del producto encontrando multiplicadores más débiles. Sus multiplicadores se ubican en un rango de -0.1 a 1.3. De Castro Fernández y Hernández de Cos (2006) encuentran un valor similar a este último a la hora de estimar el multiplicador en España.

En una contribución sumamente interesante Ilzetzki, Mendoza y Vegh (2010) estiman un SVAR para un panel de 44 países considerando las características específicas de cada país (nivel de desarrollo, apertura, régimen cambiario, deuda pública y la inversión del gobierno). Analizando sólo la respuesta del producto ante movimientos en el gasto del gobierno, en general encuentran multiplicadores de impacto muy pequeños (0.24 para países desarrollados y 0.04 para países en desarrollo) y multiplicadores acumulados mayores en países desarrollados que en desarrollo (1.04 versus $0.79 \mathrm{al}$ cabo de 24 trimestres). Un resultado muy relevante para países en desarrollo indica que el multiplicador de la inversión del gobierno es positivo, cercano a 1 en el mediano plazo, y estadísticamente diferente del multiplicador del consumo público en horizontes de pronóstico de hasta dos años. Esto indica que la composición del gasto puede jugar un papel importante en la evaluación de los efectos del estímulo fiscal en los países en desarrollo. ${ }^{16}$

Más recientemente, Auerbach y Gorodnichenko (2012) enriquece la estrategia de Blanchard y Perotti (2002) mediante la inclusión de no linealidades en el modelo que permiten estimar los multiplicadores para diferentes fases del ciclo (expansión y recesión). Sus conclusiones pueden resumirse en multiplicadores del gasto muy altos en épocas de recesión: 1.5 en un año, 1.25 en 5 año; y multiplicadores negativos en tiempos de expansión.

Con menor intensidad que para los países desarrollados se registran estudios para países en desarrollo, varios de ellos pertenecientes a América Latina. Una serie de estudios encuentran un rango amplio para el multiplicador del gasto público que va desde 0.1 para el caso de Colombia a 2.2 en Perú. ${ }^{17}$ Por último vale destacar la ausencia de la medición del multiplicador del gasto para Argentina utilizando esta metodología. Sin embargo debe mencionarse que si bien no miden dicho multiplicador Avramovich, Basso y Rezk (2006) estudian los efectos de la política fiscal argentina en el marco de un SVAR de 5 variables similar al utilizado por Perotti (2004). Encuentran un efecto desplazamiento del gasto público, lo que da indicios (en base a lo expuesto desde el punto de vista teórico) que Argentina debería presentar un multiplicador del gasto público mayor a cero pero menor a la unidad. En la misma dirección, pero haciendo uso de un modelo VAR (no estructural), Anós Casero, Cerdeiro y Trezzi (2010) encuentran un multiplicador del gasto publico en Argentina pequeño y de corta duración ${ }^{18}$, sembrando dudas sobre la efectividad del estímulo fiscal.

\subsubsection{Enfoque narrativo}

El enfoque SVAR ha sido criticado severamente en cuanto a la estrategia de identificación utilizada. Ramey (2011) basa sus críticas argumentando que diferenciar entre el momento en que se anuncian los shocks de aquel en el cual se producen como así también el tratamiento de las expectativas son dos aspectos cruciales. ${ }^{19}$ Más concretamente, los shocks que calculan los investigadores pueden ser anticipados por los agentes económicos, aunque los datos trimestrales no lo permitan. Estas anticipaciones están presentes dado que muchos cambios en la política fiscal sólo son exógenos en

\footnotetext{
${ }^{16} \mathrm{~A}$ su vez encuentran que tipos de cambio flexibles conducen a multiplicadores cercanos a cero, los multiplicadores son menores en economías abiertas y negativos en países muy endeudados.

${ }^{17}$ Véase Restrepo y Rincón (2006) Cespedes, Fornero y Gali (2011) y Sanchez Tapia y Galindo Gil (2013).

${ }^{18}$ Ante un aumento de 1 millón de pesos en el gasto, el consumo privado aumentan aproximadamente $0.40 \%$.

${ }^{19}$ Véase, por ejemplo, Ramey and Shapiro (1998), Ramey (2011).
} 
el momento de su anuncio, pero no en su aplicación efectiva. En contraposición, argumenta que estrategias narrativas pueden captar mejor las diferencias entre el momento del anuncio y la implementación de las políticas. Más recientemente, Leeper, Walker y Yang (2013) han argumentado que las noticias periodísticas deben tenerse en cuenta al calcular el efecto de la política fiscal. ${ }^{20}$

Aunque el enfoque narrativo por lo general también se basa en la estimación de un vector autorregresivo (VAR), la estrategia de identificación consiste en analizar los cambios pasados en el gasto público y argumentar cualitativamente que esos cambios pueden ser identificados como exógenos. El ejemplo tradicional de cambios exógenos es el gasto militar de Estados Unidos, ya que a menudo se argumenta que las acciones militares están totalmente no correlacionadas con otras variables macroeconómicas. Uno de los primeros trabajos para aplicar este enfoque es Ramey y Shapiro (1998) quienes utilizan una nueva medida de los shocks de gasto militar de Estados Unidos y estiman el efecto de los grandes aumentos de este gasto (por ejemplo durante los conflictos bélicos con Corea, Vietnam e Irak). Sus resultados muestran un claro efecto riqueza negativo sobre el consumo y el producto. Perotti (2007) también recurre a un análisis narrativo para realizar una comparación con sus resultados obtenidos mediante el enfoque SVAR. Al igual que en los resultados del análisis estructural, encuentra resultados que contradicen los de Ramey y Shapiro (1998), es decir, un efecto muy positivo sobre el producto, el consumo privado, la formación de capital privado y las diferentes variables de empleo.

Romer y Romer (2010) introducen nuevas medidas de shocks exógenos de la política fiscal pero por el lado de los impuestos. Analizando los principales discursos e informes de funcionarios gubernamentales evalúan la exogeneidad de los cambios impositivos. Los autores encuentran un claro efecto negativo del aumento de los impuestos sobre el producto (multiplicadores acumulados mayores a 2), la tasa de desempleo y la tasa de inflación. Los cambios endógenos parecen no tener ningún efecto significativo. Riera Crichton, Vegh y Vuletín (2012) refuerzan está idea encontrando que los cambios endógenos utilizando el enfoque SVAR de Blanchard y Perotti (2002) puede arrojar multiplicadores de los impuestos neutrales o hasta incluso expansivos. ${ }^{21}$

\footnotetext{
${ }^{20}$ Otros autores critican que los modelos SVAR no tienen en cuenta los cambios en el precio de los activos, que pueden tener una influencia considerable en los ingresos fiscales (Baum et al, 2012).

${ }^{21}$ Para un abordaje más profundo de trabajos que discuten las cuestiones de identificación puede verse Favero y Giavazzi (2007), Caldara y Kamps (2008) y Ramey (2011).
} 


\section{Metodología}

En este trabajo se estima un vector autorregresivo estructural (SVAR) siguiendo a Blanchard y Perotti (2002). Para ello se utiliza la siguiente especificación de referencia:

$$
X_{t}=A(L) X_{t-1}+U_{t}
$$

donde $X_{t} \equiv\left[g_{t}, y_{t}\right]^{\prime}$, es un vector de dos variables endógenas para el trimestre t. Específicamente las primeras diferencias de los logaritmos naturales de las series gasto público y producto bruto interno (PIB) ajustadas estacionalmente. El polinomio $A(L)$ describe la relación entre los coeficientes en cada trimestre mientras que $U_{t}=\left[u_{t}^{g}, u_{t}^{y}\right]^{\prime}$ incorpora los residuos de forma reducida.

Los coeficientes para los diferentes rezagos se calculan utilizando Mínimos Cuadrados Ordinarios (MCO), el mejor estimador lineal e insesgado en este caso (Greene, 2008) y de esas estimaciones se obtiene el vector de los residuos.

Sin embargo, para poder establecer conclusiones sobre la causalidad dentro de la política fiscal, y dado que los residuos de la forma reducida no son muy informativos en sí mismo, se deben recuperar los shocks estructurales de los residuos. Estos shocks estructurales no se encuentran correlacionados mutuamente y representan el principal punto de interés aquí. Su obtención se realiza por descomposición de los residuos de forma reducida de la siguiente manera:

$$
\begin{aligned}
& u_{t}^{g}=a_{1} u_{t}^{y}+e_{t}^{g} \\
& u_{t}^{y}=a_{2} u_{t}^{g}+e_{t}^{y}
\end{aligned}
$$

donde $u_{t}^{g}$ y $u_{t}^{y}$ son los movimientos inesperados en el gasto y el producto. Por su parte $e_{t}^{g}$ y $e_{t}^{y}$ son los shocks estructurales que se desean estimar ya que incluyen, entre otros, los shocks de oferta y demanda, los cambios de tecnología o en la política monetaria y fiscal. Así, capturan la parte de $U_{t}$ producida por factores exógenos, es decir, que no depende de la evolución de la economía. Las ecuaciones (2) y (3) pueden reescribirse del siguiente modo como matrices:

$$
\left(\begin{array}{cc}
1 & -a_{1} \\
-a_{2} & 1
\end{array}\right) * U_{t}=\left(\begin{array}{ll}
1 & 0 \\
0 & 1
\end{array}\right) * E_{t}
$$

donde $E_{t} \equiv\left[e_{t}^{g}, e_{t}^{y}\right]^{\prime}$.

Ahora bien, sobre el parámetro $a_{1}$ se presenta el supuesto fundamental respecto a la estrategia de identificación de los shocks fiscales. Dado el problema de endogeneidad mencionado anteriormente y que afecta a las variables del modelo, este parámetro pueden contener dos tipos de información: cambios endógenos en el gasto debido a cambios en el PIB y cambios exógenos de la política fiscal como respuesta a movimientos extraordinarios inesperados en el producto. Dado que se utilizan datos trimestrales, se asume que el segundo canal no es posible dentro de un mismo trimestre, en cuanto a que un gobierno no es capaz de reaccionar rápida ni automáticamente al ciclo económico. Además, las respuestas políticas también demoran más de un trimestre dado que por ejemplo una nueva decisión de gasto generalmente tiene que pasar ante el Poder Legislativo y requiere su aceptación para ser activado. Este último punto implica que $a_{1}=0$. De esta manera, la hipótesis 
implícita aquí presente es que los shocks de política fiscal causan efectos cuando son implementados y no cuando son anunciados.

Por último puede estimarse el SVAR incorporando todas las restricciones antes mencionadas y obtener las correspondientes funciones de impulso respuesta que describen la reacción de las variables explicadas en el sistema ante cambios en los errores estructurales (los cambios exógenos).

En base a los valores obtenidos de las funciones impulso respuestas, se sigue la práctica tradicional para la construcción de los multiplicadores del gasto (tanto de impacto como acumulativo) multiplicando éstas por el valor medio del ratio producto/gastos para el período considerado. ${ }^{22}$

Se define como multiplicador de impacto $\frac{d Y_{t+k}}{d G_{t}}=\frac{d \log Y_{t+k}}{d \log G_{t}} \cdot \frac{Y_{t+k}}{G_{t}}$. El multiplicador acumulativo será entonces $\frac{\sum_{j=1}^{k} d Y_{t+j}}{\sum_{j=1}^{k} d G_{t+j}}$.

\section{Datos}

Para estimar los modelos SVAR propuestos en este trabajo se utilizan datos trimestrales para el período que abarca desde el primer trimestre de 1993 hasta el cuarto de 2012.

Las variables macroeconómicas argentinas consideradas son: los gastos del Sector Público Nacional y el producto bruto interno (PBI). ${ }^{23}$ Adicionalmente a la hora de analizar los mecanismos de transmisión del gasto se utilizan las series de consumo privado, inversión privada y exportaciones netas.

Dado que se cuenta con la apertura del gasto público según su clasificación económica se considerará luego la distinción entre gasto en consumo (o corriente) y gasto en capital. En relación al primer rubro se considerarán el gasto en consumo y operaciones que contiene mayormente el componente salarial, el gasto en prestaciones a la seguridad social que capta el pago de jubilaciones y las transferencias corrientes. No se hace uso de la partida rentas de la propiedad que representa pagos de interese y es menos plausible (a priori) que afecten la demanda de consumo. El gasto en capital contiene el gasto en inversión real directa y las transferencias de capital que realiza el gobierno central. De estas partidas puede suponerse que afecten el producto a través de las decisiones de inversión del Sector Público y que pueden generar sinergias con el sector privado. Por ejemplo, la construcción de una ruta puede ser determinante para la instalación de un establecimiento productivo.

Las series fiscales se obtuvieron del Esquema Ahorro Inversión Financiamiento (AIF) del Sector Público Nacional publicadas por el Ministerio de Economía de la Nación Argentina (MECON). El PBI y el resto de los agregados macroeconómicos provienen del Instituto Nacional de Estadística y Censos (INDEC).

Las variables fueron deflactadas por el índice de precios implícitos del PIB de Argentina para expresarlas en términos reales, desestacionalizadas siguiendo el procedimiento Census X12 multiplicativo y se les calculó el logaritmo natural. Las pruebas de raíz unitaria (Dickey-Fuller aumentada) se

\footnotetext{
${ }^{22}$ En muchos casos se utiliza el valor mediano en lugar del promedio del ratio producto/gasto para evitar la influencia de valores extremos. Se aclara que los resultados obtenidos en este trabajo son robustos a la utilización de dicha medida alternativa.

${ }^{23}$ Debe destacarse que desde 2007 en la definición de Gasto Corriente (y por lo tanto en el Gasto Total) no se contabilizan las transferencias por coparticipación a provincias. Hasta Diciembre de 2006, en el esquema AhorroInversión-Financiamiento, tanto en los ingresos corrientes como en los gastos corrientes se incluían, respectivamente, los recursos destinados a ser coparticipados a las provincias y las transferencias que se les enviaba bajo este concepto.
} 
realizaron para todas las variables endógenas y no pueden rechazar la hipótesis nula de existencia de una raíz unitaria, es decir, las variables no son estacionarias. Ante ello y para evitar posibles problemas de relaciones espurias se le calculó la primera diferencia a las variables para su inclusión en la estimación de los modelos ${ }^{24}$. El número óptimo de rezagos para cada especificación estimada se determino utilizando el criterio de información de Akaike.

\section{Resultados}

\subsection{Efectos del gasto público sobre el producto}

Como primer paso en este trabajo se presenta el multiplicador del gasto total del gobierno central de Argentina obtenido a través de un SVAR entre dicho gasto y el producto. Se puede apreciar en la Figura 1 que el gasto presenta un multiplicador bajo, con un valor nulo en el impacto y que estadísticamente no difiere de cero. ${ }^{25}$ Esto sugiere que los aumentos en el gasto del gobierno pueden ser bastante lentos en afectar la actividad económica y deja planteado interrogantes sobre la utilidad de una política fiscal discrecional para propósitos de estabilización de corto plazo.

De cualquier modo, focalizar únicamente en el multiplicador de impacto, puede ser engañoso dado que los paquetes de estímulo fiscal pueden aplicarse a lo largo del tiempo y pueden existir retrasos en la respuesta de la economía. Así, se observa un multiplicador significativo desde el punto de vista estadístico y que acumula un valor de 0.18 al cabo de 2 años ( 8 trimestres), indicando que en dicho horizonte por cada $\$ 1$ gastado por el gobierno el producto se incrementa $\$ 0.18$. Esto refleja la evidencia de efecto desplazamiento sobre la producción por parte del gasto realizado por el Sector Público Nacional.

Figura 1. Multiplicador acumulado del gasto público total del Gobierno Central de Argentina. Variables en diferencias.

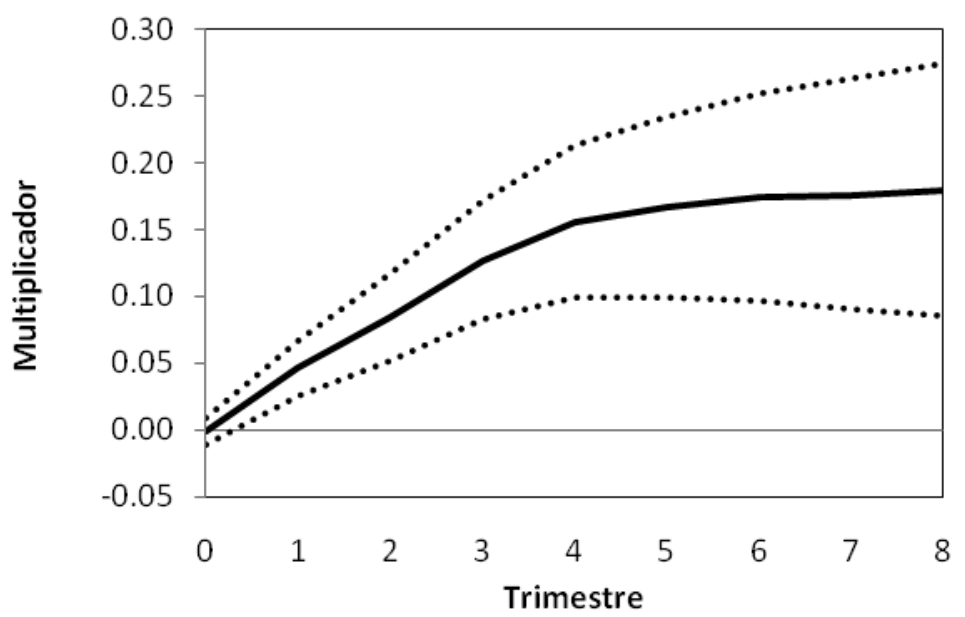

Este multiplicador de pequeña magnitud se alinea con resultados hallados por investigaciones previas para países en desarrollo (Ilzetzki, Mendoza y Vegh; 2010) y la existencia de efecto des-

\footnotetext{
${ }^{24}$ Un procedimiento alternativo aquí consiste en la inclusión de una tendencia determinísitca o estocástica en la estimación del modelo.

${ }^{25}$ Las líneas punteadas representan los intervalos de confianza de $+/-1$ desvío estándar.
} 
plazamiento del gasto público refuerza el resultado encontrado previamente en la literatura para Argentina (Avramovich, Basso y Rezk; 2006). Adicionalmente es consistente con el multiplicador esperado dada la evidencia que sostiene una política fiscal procíclica en Argentina. La imposibilidad de no poder expandir el gasto en las recesiones (ser contracíclicos) hace que se dificulte impulsar la economía en los malos momentos y generar un fuerte impacto positivo sobre el nivel de actividad.

A continuación y producto de contar con la información desagregada según la clasificación económica del gasto, se diferencia el multiplicador del gasto público según se trate de gasto en consumo o gasto en capital (o inversión pública). La Figura 2 muestra un multiplicador para este último consistentemente mayor que para el gasto en consumo, tanto en el impacto ( $\$ 0.3$ versus $\$ 0$ ) como en el acumulado en un horizonte de 2 años ( $\$ 1.03$ versus $\$ 0.18$ ). Nótese que el multiplicador del gasto en consumo (o gasto corriente) es prácticamente similar al del gasto total producto de que dicha clasificación representa más del $90 \%$ del gasto total durante toda la muestra utilizada. ${ }^{26}$

Los resultados muestran la enorme importancia de dicha diferenciación en cuanto el gasto el consumo tiene efectos moderados sobre el producto, mientras que la inversión pública tiene efectos macroeconómicos mucho más expansivos.

Figura 2. Multiplicador acumulado del gasto público en consumo y en capital del Gobierno Central de Argentina. Variables en diferencias.

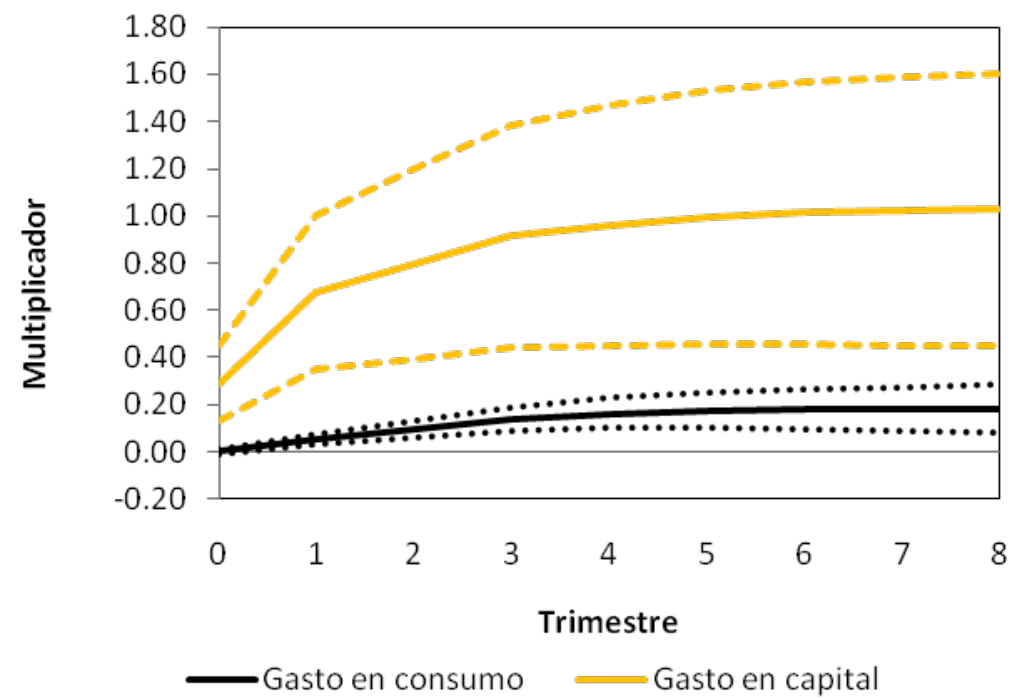

\subsection{Componentes del producto y mecanismos de transmisión}

Una vez estimados los multiplicadores sobre el producto, se realizaron estimaciones para el resto de los componentes de la demanda agregada con el fin de explorar la dinámica del gasto público o, dicho de otro modo, sus mecanismos de transmisión. Específicamente se estimaron los mismos SVAR con dos variables pero en lugar del producto se utilizó el consumo privado, la inversión privada y las exportaciones netas.

\footnotetext{
${ }^{26}$ En el Anexo a este trabajo se presenta la composición del gasto del Sector Público Nacional para el período bajo análisis.
} 
La Figura 3 muestra los resultados para cada componente diferenciando, al igual que en la sección anterior, entre consumo y capital. Un primer resultado que puede apreciarse consiste en que el gasto público tiene un multiplicador positivo y estadísticamente significativo sobre el consumo y la inversión privada mientras que sobre las exportaciones netas el multiplicador es negativo aunque no mantiene significatividad estadística.

Analizando puntualmente cada componente se observa que sobre el consumo privado (Panel A) se repite, al igual que en el caso del producto, la presencia de un multiplicador del gasto en capital mayor que el del gasto en consumo. Al cabo de 2 años el primero presenta un valor de $\$ 1.15$ mientras que el segundo acumula $\$ 0.12$. El patrón anterior se sostiene también para el caso de la inversión privada (Panel B) aunque con multiplicadores de menor magnitud. El multiplicador del gasto en capital es $\$ 0.82$ mientras que el del gasto en consumo se ubica en $\$ 0.07$.

Sin arrojar significatividad estadística, los multiplicadores del gasto sobre las exportaciones netas son negativos. En el caso del gasto en consumo se observa una caída de $\$ 0.05$ a los dos años, mientras que la caída generada por el gasto en capital es de $\$ 0.68$ (Panel C).

Así, es deseable destacar que al igual que se vio en los efectos sobre el producto la composición del gasto es un tema bien relevante a la hora de analizar sus efectos sobre la actividad económica. Otro punto, no menos importante, viene dado por el hecho de que los multiplicadores del gasto en capital sobre la inversión privada son positivos producto de las sinergias que se generan entre estos dos rubros. Así, se pone de manifiesto la importancia de pensar el sector público y el privado como complementos más que como sustitutos (o rivales). 
Figura 3. Multiplicador acumulado del gasto público en consumo y en capital del Gobierno Central de Argentina sobre el consumo privado, inversión privada y exportaciones netas.

Variables en diferencias.

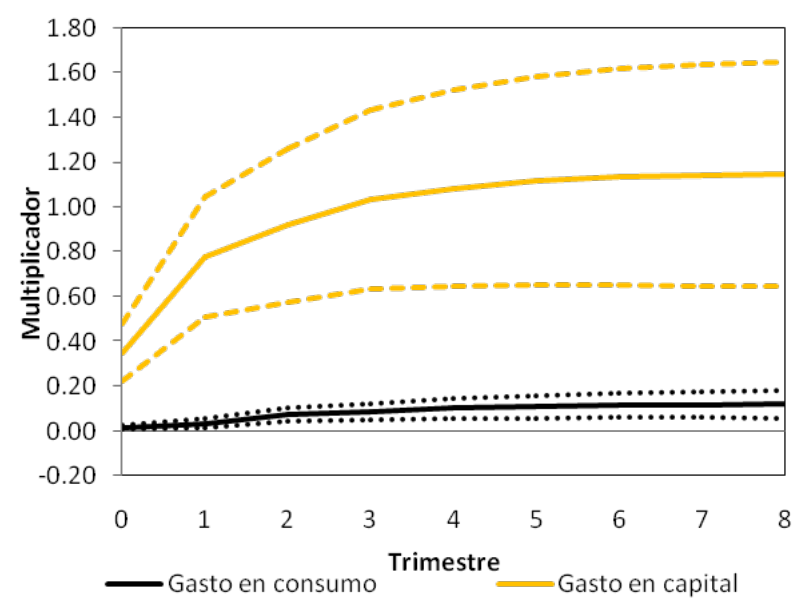

Panel A. Efecto multiplicador del gasto público sobre el consumo privado

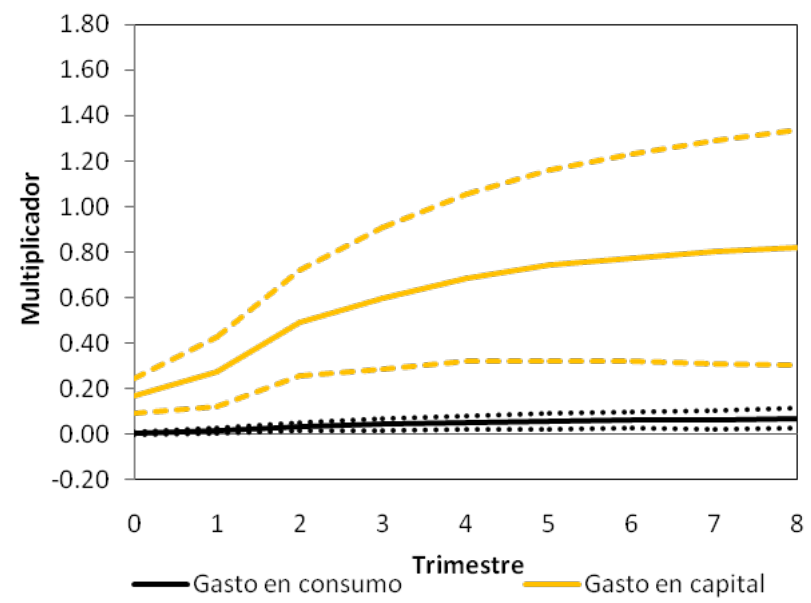

Panel B. Efecto multiplicador del gasto público sobre la inversión privada

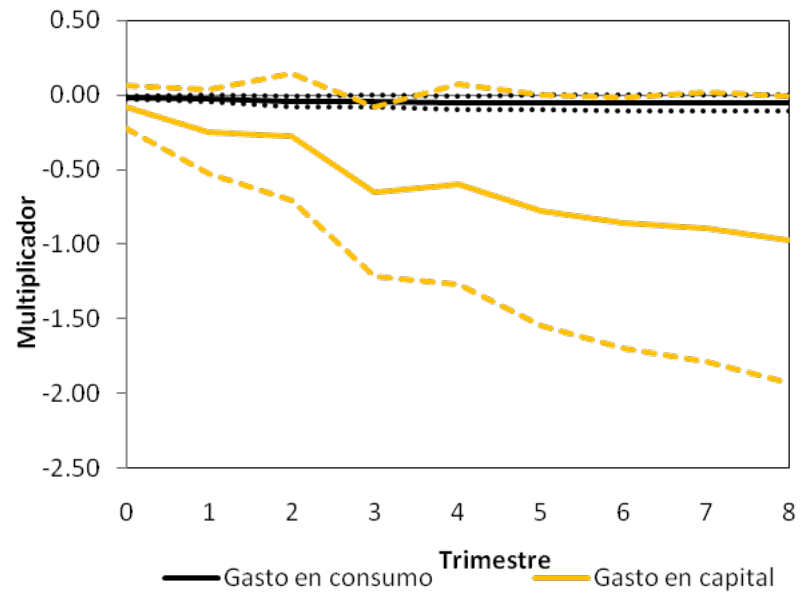

Panel C. Efecto multiplicador del gasto público sobre las exportaciones netas 
Dados estos resultados, para indagar aún mas sobre la transmisión del gasto se estimó un SVAR entre consumo privado e inversión privada dado que son los dos componentes de la demanda que reaccionan positivamente y con soporte estadístico a movimientos en el gasto público. Los efectos se estimaron en ambos sentidos con el objetivo de analizar si el consumo reacciona más ante movimientos en la inversión o si por el contrario es la inversión quién presenta mayor reacción ante movimientos en el consumo. La Figura 4 soporta la primera de éstas hipótesis. El efecto es más fuerte cuando se analiza la dirección desde inversión hacia consumo. Esto, vinculado al multiplicador del gasto público en capital sobre la inversión privada hallado anteriormente, refuerza la idea de pensar en la existencia de cierto grado de complementariedad entre la inversión pública y la privada, que a su vez genera un efecto positivo sobre el consumo privado.

Figura 4. Multiplicadores acumulados resultantes de un SVAR entre consumo privado e inversión privada. Variables en diferencias.

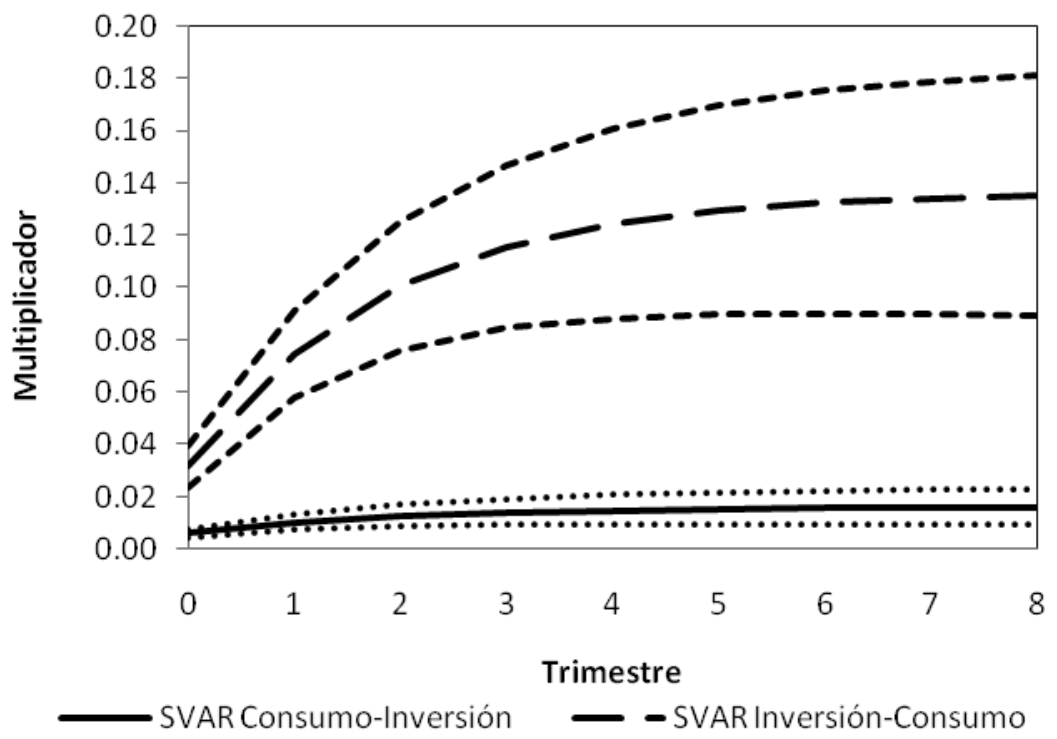

\section{Extensión al caso de 3 variables}

Hasta ahora el enfoque utilizado en este estudio se ha basado en la estimación de SVARs con dos variables. Sin embargo resulta interesante ver si los efectos difieren o se mantienen ante la estimación de un vector de tres variables endógenas. Para explorar esta pregunta, se volvió a estimar la ecuación (1), esta vez con $X_{t} \equiv\left[g_{t}^{c a p}, g_{t}^{c o n}, y_{t}\right]^{\prime}$, donde $g_{t}^{c a p}$, es el gasto en capital, $g_{t}^{c o n}$ es el gasto en consumo e $y_{t}$ representa el producto. Siguiendo a Perotti (2004) e Ilzetzki, Mendoza y Vegh (2010) se ordena primero al gasto en capital primero y luego al de consumo, aunque los resultados no se alteran si el orden se invierte.

Estas estimaciones se controlan por el gasto en consumo del gobierno, pero se sigue a Perotti (2004) e Ilzetzki, Mendoza y Vegh (2010) al estimar el multiplicador de los cambios exógenos en el gasto en capital del gobierno, que impiden respuestas endógenas de dicho gasto y el producto al gasto en consumo del gobierno. Esto se realiza estimando el sistema completo con las tres 
variables endógenas, fijando todos los valores de $g_{t}^{c o n}=0$ en los pronósticos de $g_{t}^{c a p}$ e $y_{t}$ al estimar las funciones impulso respuesta. Esto se hace para asegurar que no se confunda la respuesta del producto al gasto en capital con el gasto en consumo del gobierno dado que ambas variables de gasto covarían fuertemente.

Los resultados se presentan en la Figura 5. Se aprecia al igual que en el caso de dos variables que los multiplicadores generados por el gasto en capital son mayores que los del gasto en consumo en todos los casos. Un aumento en $\$ 1$ del primero genera $\$ 1.90$ adicionales de producto, mientras que el mismo incremento en el gasto en consumo hace lo propio en $\$ 0.16$ (Panel A). Un punto a tener en cuenta es que a diferencia del caso de dos variables donde el multiplicador del gasto en capital era consistentemente mayor que el de consumo, aquí el efecto en el impacto de ambos tipos de gastos es prácticamente nulo y estadísticamente no significativo. Sin embargo al cabo de un horizonte de dos años las diferencias son apreciables y significativas.

Adicionalmente los multiplicadores sobre el consumo privado y la inversión privada son positivos, dando más soporte a la complementariedad entre el sector público y el privado. En el caso del consumo privado (Panel B) se tiene un multiplicador de $\$ 1.18$ para el gasto en capital mientras que el de consumo se ubica en $\$ 0.06$. Para la inversión privada el multiplicador alcanza valores de $\$ 0.66$ y $\$ 0.03$ respectivamente (Panel C). Por último, menos clara es la reacción de las exportaciones netas, donde sin arrojar significatividad estadística el multiplicador del gasto en consumo es negativo en $\$ 0.03$ y el del gasto en capital también, con un valor de $-\$ 0.18$. 

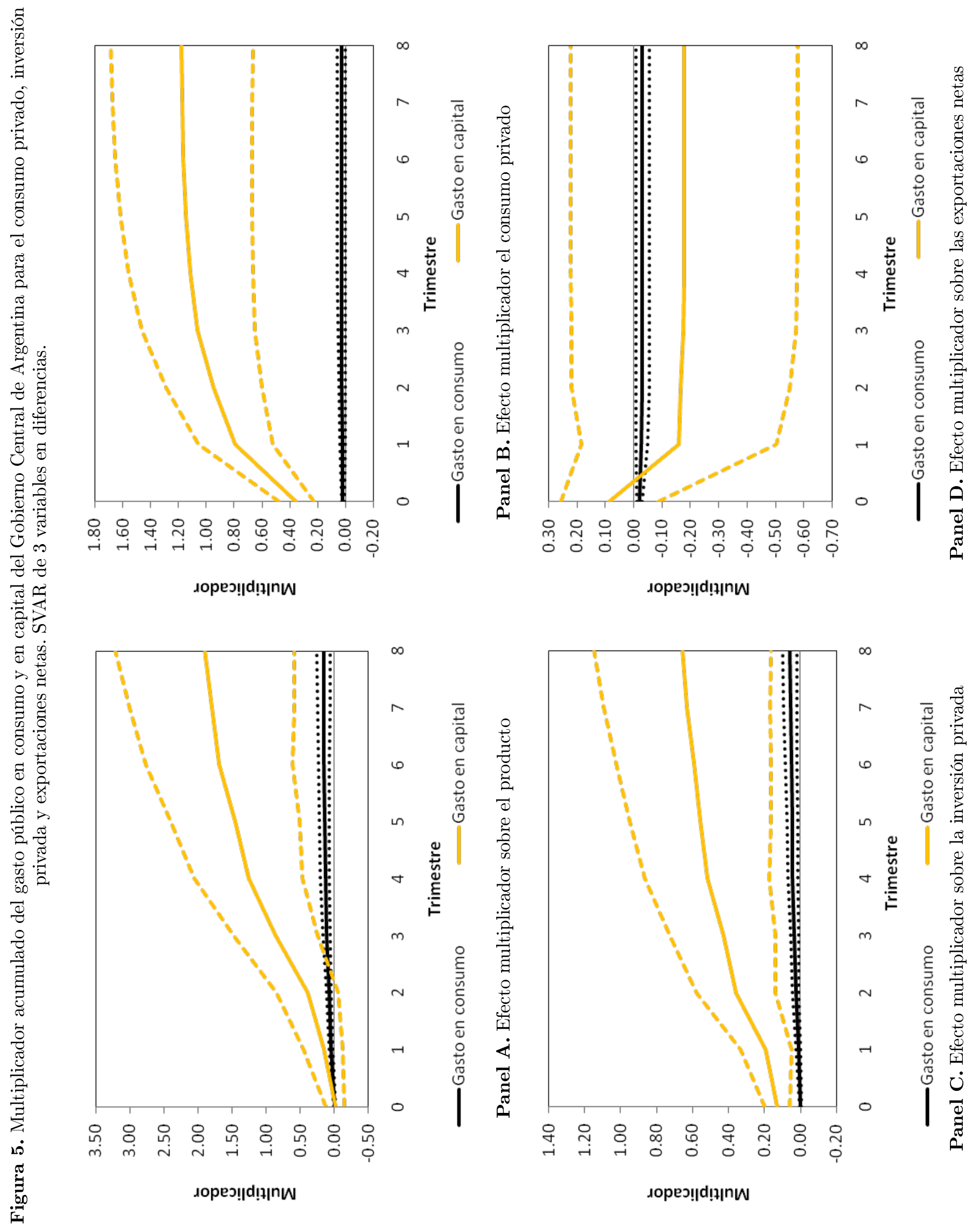

ıореग!|d!ınw

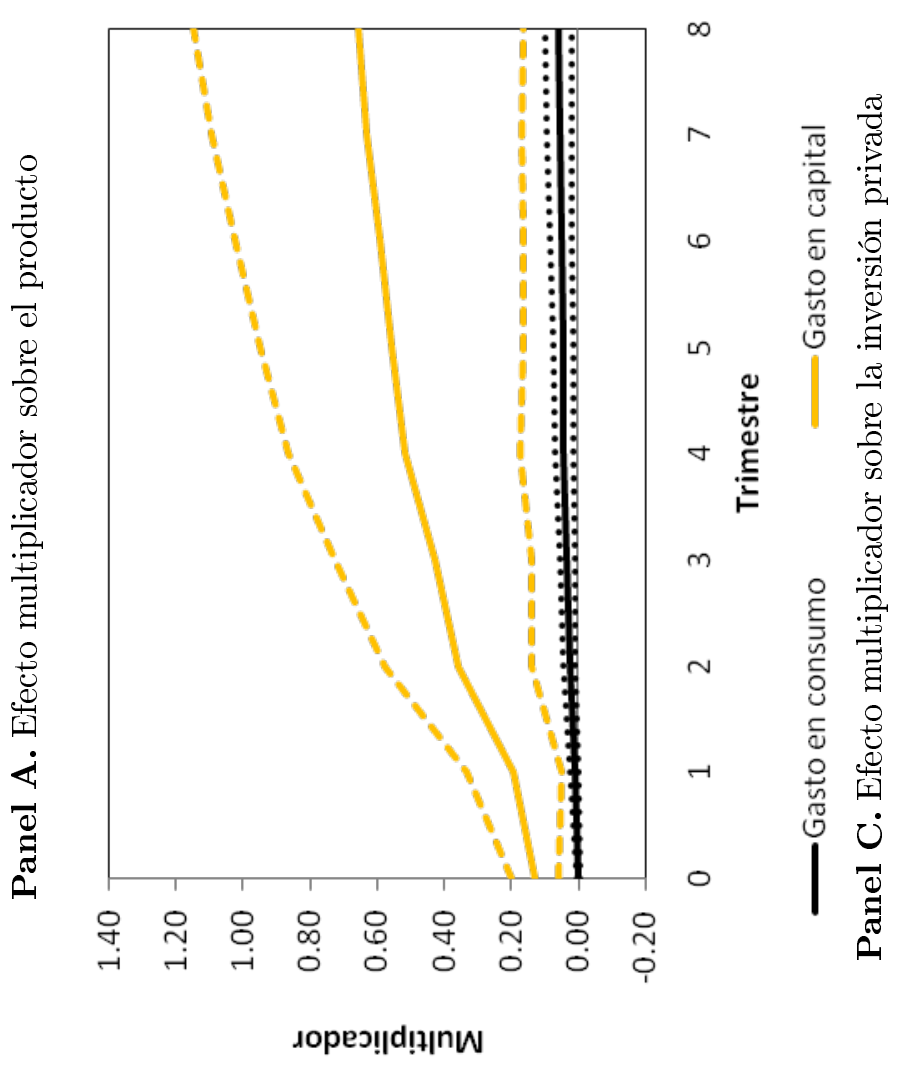




\section{Comentarios finales}

La última crisis económica acontecida a nivel mundial ha forzado a que muchos países implementaran paquetes de estímulo o medidas de austeridad fiscal y como consecuencia han proliferado los estudios empíricos con el fin de echar luz sobre una de las preguntas centrales en la política macroeconómica de los últimos años: ¿Cuál es el efecto del gasto del gobierno sobre la actividad económica?

Si bien la mayoría de los estudios pusieron el foco sobre para países desarrollado, diversos estudios se han centrado en países en desarrollo con la excepción de Argentina. En este trabajo se propuso contribuir a la literatura estimando por primera vez el multiplicador del gasto del Sector Público Nacional en Argentina utilizando la metodología SVAR. Al mismo tiempo, producto de contar con la clasificación económica del gasto, se diferenció el multiplicador del gasto en consumo en contraposición al gasto en capital para indagar si la composición del gasto es un determinante de los efectos que éste genera sobre el producto en Argentina.

Los resultados sostienen que el multiplicador del gasto público en Argentina, al igual que otros países en desarrollo, es bajo y se sitúa en $\$ 0.18$ al cabo de 8 trimestres. Esto pone de manifiesto la presencia de efectos desplazamiento sobre la producción y es consistente con el resultado esperado dada la tradicional prociclicidad de la política fiscal del país.

Además el multiplicador del gasto en consumo se encuentra muy por debajo del multiplicador del gasto en capital. El primero alcanza también de $\$ 0.18$ y esto se explica dado que el gasto en consumo representa más del $90 \%$ del gasto total. El multiplicador del gasto en capital es sistemáticamente mayor a la unidad mostrando la enorme importancia de la composición del gasto en cuanto a que el gasto el consumo tiene efectos moderados sobre el producto, mientras que la inversión pública tiene efectos macroeconómicos mucho más expansivos. Otro punto, no menos importante, viene dado por el hecho de que los multiplicadores del gasto en capital sobre la inversión privada son positivos producto de las sinergias que se generan entre estos dos rubros. Así, se pone de manifiesto la importancia de pensar el sector público y el privado como complementos más que como sustitutos (o rivales).

De este modo, los resultados encontrados plantean interrogantes sobre el grado de efectividad del gasto público sobre la actividad económica como así también sugieren que debe ponerse atención en cómo se compone dicho gasto. Se refuerza a su vez la idea de que Argentina se beneficiaría de una mejor articulación entre el sector público y el privado, y del abandono de políticas fiscales procíclicas. La incapacidad de ahorrar en tiempos de bonanza aumenta en gran medida la probabilidad de que los malos tiempos se conviertan en crisis fiscales hechas y derechas. 


\section{Referencias}

[1] Anos-Casero, Paloma, Diego Cerdeiro y Ricardo Trezzi, 2010. "Estimating the fiscal multiplier in Argentina". , The World Bank, Policy Research Working Paper Series 5220.

[2] Auerbach, Alan y Yuriy Gorodnichenko, 2011. "Fiscal multipliers in recession and expansion". NBER Working Paper No. 17447.

[3] Auerbach, Alan y Yuriy Gorodnichenko 2012. "Measuring the output responses to fiscal policy". American Economic Journal: Economic Policy, 4(2):1

[4] Avramovich, Maria C., Martin Basso y Ernesto Rezk, 2006. "Dynamic effects of fiscal shocks upon diverse macroeconomic variables: a structural VAR analysis for Argentina". XVIII Seminario Regional de Política Fiscal, CEPAL.

[5] Barro, Robert, 1981. "Output effects of government purchases". Journal of Political Economy, Vol. 89, pp. 1086-1121.

[6] Barro, Robert. y Charles Redlick, 2011. "Macroeconomic effects from government purchases and taxes". The Quarterly Journal of Economics, Vol. 126, pp. 51-102.

[7] Baum, Anja, Marcos Poplawski-Ribeiro y Anke Weber, 2012. "Fiscal multipliers and the state of the economy". IMF Working Papers 12/286.

[8] Baxter, Marianne y Robert King, 1993. "Fiscal policy in general equilibrium". American Economic Review, Vol. 83, pp. 315-334.

[9] Blanchard, Olivier y Roberto Perotti, 2002. "An empirical characterization of the dynamic effects of changes in government spending and taxes on output". The Quarterly Journal of Economics, Vol. 117, pp. 1329-1368.

[10] Braun, Miguel y Luciano Di Gresia, 2003. "Towards Effective Social Insurance in Latin America: The Importance of Countercyclical Fiscal Policy". Inter-American Development Bank. Research Department. Working Paper \#487.

[11] Caldara, Dario y Christophe Kamps, 2008. "What are the effects of fiscal policy shocks? A VAR-based comparative analysis". European Central Bank. Working Paper Series 0877.

[12] Cerda, Rodrigo, Hermann González y Luis F. Lagos, 2003. “¿Es efectiva la política fiscal? Evidencia para una economía emergente". Instituto de Economía. Pontifica Universidad Católica de Chile. Documento de Trabajo № 249.

[13] Céspedes , Luis F., Jorge A. Fornero y Jordi Galí, 2011. "Non-Ricardian Aspects of Fiscal Policy in Chile". Journal Economía Chilena (The Chilean Economy), Central Bank of Chile, vol. 14(2), pp. 79-107.

[14] Cwik, Tobias y Volker Wieland, 2009. "Keynesian government spending multipliers and spillovers in the euro area". CEPR Discussion Papers 7389.

[15] De Castro Fernández, Francisco y Pablo Hernández de Cos, 2006. "The economic Effects of Exogenous fiscal Shocks in Spain. A SVAR approach". European Central Bank. Working Paper $\mathrm{N}^{\circ} 647$. 
[16] Díaz Frers, Luciana, 2010. "Los caminos paralelos al laberinto de la coparticipación". CIPPEC Documento de Políticas Públicas / Recomendación №ำ.

[17] Favero, Carlo y Francesco Giavazzi, 2007. "Debt and the effects of fiscal policy". NBER Working Paper No. 12822.

[18] Favero, Carlo y Francesco Giavazzi, 2010. "Reconciling VAR-based and the narrative measures of the tax multiplier". CEPR Discussion Papers 7769.

[19] Frankel, Jeffrey A., Carlos A. Végh y Guillermo Vuletin, 2011. "On Graduation from Fiscal Procyclicality". NBER Working Papers 17619.

[20] Galindo Gil, Hamilton y William Sánchez Tapia, 2013. "Multiplicadores Asimétricos del Gasto Público y de los Impuestos en el Perú". Ministerio de Economía y Finanzas de la República del Perú.

[21] Gavin, Michael y Roberto Perotti, 1997. "Fiscal Policy in Latin America". NBER Macroeconomics Annual

[22] Gordon, Robert y Robert Krenn, 2010. "The end of the great depression 1939-41: Policy contributions and fiscal multipliers". NBER Working Paper No. 16380.

[23] Greene, William. H, 2008. "Econometric Analysis". 5th Edition. New Delhi, India: Pearson Education.

[24] Hall, Robert, 2009. "By how much does GDP rise if the government buys more output?" Brookings Papers in Economic Activity, Vol. 2, pp. 183-231.

[25] Ilzetzki, Ethan, Enrique Mendoza y Carlos Vegh, 2010. "How big (small?) are fiscal multipliers?" NBER Working Paper No. 16479.

[26] Ilzetzki, Ethan, 2011. "Fiscal policy and debt dynamics in developing countries". London School of Economics. Mimeo

[27] Kaminsky, Graciela, Carmen M. Reinhart y Carlos A. Vegh, 2004, "When It Rains It Pours: Procyclical Capital Flows and Macroeconomic Policies". NBER Macroeconomics Annual.

[28] Leeper, Eric M., Todd B. Walker y ShuChun Susan Yang, 2013. "Fiscal foresight and information flows". Econometrica, 81(3):1115.

[29] Mankiw, Gregory y Mark P. Taylor, 2008. "Macroeconomics". European Edition. Worth Publishers. New York.

[30] Melgarejo, Karl y Waldo Mendoza, 2008. "La Efectividad De La Política Fiscal En El Perú: 1980-2006". Pontificia Universidad Católica del Perú. Documento de trabajo 2008-262.

[31] Mountford, Andrew y Harald Uhlig, 2009. "What are the effects of fiscal policy shocks?". Journal of Applied Econometrics, Vol. 24, pp. 960-992.

[32] Perotti, Roberto, 2004. "Estimating the Effects of Fiscal Policy in OECD Countries". European Central Bank. Working Paper $\mathrm{N}^{\circ} 168$.

[33] Perotti, Roberto, 2007. "In Search of the Transmission Mechanism of Fiscal Policy". NBER Working Paper $N^{\circ} 13143$. 
[34] Porto, Alberto, 2004. "Disparidades regionales y federalismo fiscal", UNLP, EDULP , pp 11146.

[35] Ramey, Valerie y Matthew Shapiro, 1998. "Costly capital reallocation and the effects of government spending." Carnegie-Rochester Conference Series on Public Policy, Vol. 48, pp. 145-194.

[35] Ramey, Valerie, 2011. "Identifying government spending shocks: It's all in the timing". The Quarterly Journal of Economics, Vol. 126, pp. 1-50.

[36] Restrepo, Jorge y Hernan Rincón, 2006. "Identifying fiscal policy shocks in Chile and Colombia". Central Bank of Chile. Working Papers N 370 .

[37] Riera-Crichton, Daniel, Carlos Vegh y Guillermo Vuletin, 2012. "Tax Multipliers: Pitfalls in Measurement and Identification". NBER Working Paper $N^{\circ} 18497$.

[38] Romer, Christina y David Romer, 2010. "The macroeconomic effects of tax changes: Estimates based on a new measure of fiscal shocks". American Economic Review, Vol. 100, pp. 763-801.

[39] Spilimbergo, Antonio, Steve Symansky y Martin Schindler, 2009. "Fiscal multipliers". IMF Staff Position Notes No. 09/11.

[40] Talvi, Ernesto y Carlos Vegh, 2005. "Tax Base Variability and Procyclical Fiscal Policy". Journal of Development Economics, Vol. 78(1), pp. 156-190 
Axexo. Gasto Total del Sector Publico Nacional No Financiero. Participaciones del gasto en consumo (Corriente) y en capital. 1993-2012 y subperíodos.

\begin{tabular}{|c|c|c|c|c|c|}
\hline Gasto & Todo el período 1993-2012 & $1993-1997$ & $1998-2002$ & $2003-2007$ & $2008-2012$ \\
\hline \hline Corriente & $90.10 \%$ & $92.00 \%$ & $93.90 \%$ & $89.00 \%$ & $88.10 \%$ \\
\hline Capital & $9.90 \%$ & $8.00 \%$ & $6.10 \%$ & $11.00 \%$ & $11.90 \%$ \\
\hline Total & $100 \%$ & $100 \%$ & $100 \%$ & $100 \%$ & $100 \%$ \\
\hline & & $1993-2002$ & $2003-2012$ \\
\hline \multicolumn{2}{|c|}{ Corriente } & $93.00 \%$ & \multicolumn{2}{c|}{$88.50 \%$} \\
\hline & Capital & $7.00 \%$ & $11.50 \%$ \\
\hline & Total & $100 \%$ & \multicolumn{2}{c|}{$100 \%$} \\
\hline
\end{tabular}

